\title{
Frontières
}

\section{Les besoins d'apprentissage, les valeurs et les croyances des infirmières concernant la douleur chronique chez la personne âgée}

\author{
Maryse L. Savoie et Sylvie Le May
}

Volume 17, numéro 2, printemps 2005

Surtout, ne pas souffrir

URI : https://id.erudit.org/iderudit/1073490ar

DOI : https://doi.org/10.7202/1073490ar

Aller au sommaire du numéro

Éditeur(s)

Université du Québec à Montréal

ISSN

1180-3479 (imprimé)

1916-0976 (numérique)

Découvrir la revue

Citer cet article

Savoie, M. L. \& Le May, S. (2005). Les besoins d'apprentissage, les valeurs et les croyances des infirmières concernant la douleur chronique chez la personne âgée. Frontières, 17(2), 59-65. https://doi.org/10.7202/1073490ar
Résumé de l'article

La présente étude exploratoire et descriptive a pour but d'évaluer les besoins d'apprentissage, les valeurs et les croyances des infirmières concernant le soulagement de la douleur chez les personnes âgées en vue d'élaborer un programme de formation sur mesure. Les infirmières ont rapporté des valeurs solides tel que donner des soins visant le confort, la qualité de vie et le respect des volontés du patient. 


\section{Les besoins d'apprentissage, les valeurs et les croyances des infirmières concernant la douleur chronique chez la personne âgée}

\section{Résumé}

La présente étude exploratoire et descriptive a pour but d'évaluer les besoins d'apprentissage, les valeurs et les croyances des infirmières concernant le soulagement de la douleur chez les personnes âgées en vue d'élaborer un programme de formation sur mesure. Les infirmières ont rapporté des valeurs solides tel que donner des soins visant le confort, la qualité de vie et le respect des volontés du patient.

Mots clés: Douleur - personne âgée infirmière - besoin d'apprentissage.

\section{Abstract}

The purpose of this study is to evaluate nurses, learning needs, values and beliefs regarding pain management of the elderly in order to develop a customized training program. Regarding values, nurses reported sound values like caring for the comfort, quality of life and the respect of their patient wishes.

Key-words: Nurses - learning needs values and beliefs regarding chronic pain in the elderly.

\section{Maryse L. Savoie, inf., M.Sc. Hôpital Sainte-Anne.}

Sylvie Le May, inf., Ph.D.

Université McGill et Université de Montréal,

Le Guide des soins de fin de vie aux ânés (2000) définit la douleur comme une expérience sensorielle ou psychique consécutive à des stimuli sensoriels et modifiée par la mémoire, les attentes et les émotions du sujet. Par ailleurs, McCaffery (1989) met l'accent sur l'aspect subjectif de la douleur et affirme que «la douleur est ce que la personne dit qu'elle est, et existe dans la mesure où la personne dit qu'elle existe».

Bien que la douleur ne soit pas liée à l'âge, il existe chez les personnes âgées une grande prévalence de maladies chroniques pouvant causer de la douleur (Marzinski, 1991). La douleur chronique est le troisième problème de santé d'importance aux ÉtatsUnis (Epps, 2001). Une étude récente (Fox et al., 1999) indique que $49 \%$ à $83 \%$ des bénéficiaires en soins prolongés souffrent de douleurs. Ces résultats sont similaires à ceux d'autres études (Bowling et Browne, 1991; Ferrell et al., 1990) qui ont estimé des taux de prévalence de la douleur chez les bénéficiaires en soins prolongés variant de 45 à $80 \%$. Les conséquences de la douleur chronique non traitée peuvent être multiples sur la santé des personnes âgées: difficulté à la mobilisation, insomnie, dépression, diminution de l'activité sociale, malnutrition, altération des fonctions mentales, polypharmacologie et chute (Closs, 1994; Ferrell et al., 1990; Ferrell, 1991; Marzinski, 1991). Toutes ces conséquences peuvent altérer sérieusement la qualité de vie des personnes âgées.
Closs (1994) mentionne que la douleur chez les personnes âgées est un phénomène négligé. L'étude de Ferrell et al. (1990) a démontré que $71 \%$ des bénéficiaires de centres de soins prolongés éprouvaient de la douleur de façon intermittente ou continue. Parmi ces personnes, seulement $15 \%$ avaient reçu un analgésique dans les 24 heures précédentes. Même si la plupart de ces bénéficiaires possédaient une prescription d'acétaminophène, seulement $10 \%$ de ces prescriptions étaient prescrites régulièrement. Ces données reflètent la piètre qualité des pratiques cliniques concernant le soulagement de la douleur chez les personnes âgées (Ryan et al., 1994).

Une autre étude réalisée en 1990 (Lindley et Dalton) a démontré que malgré les effets négatifs largement reconnus de la douleur, $60 \%$ des bénéficiaires hospitalisés et $83 \%$ des bénéficiaires à domicile recevaient leur analgésique moins fréquemment que prescrit. Dans un éditorial rédigé en 1987 pour le journal Pain, Melzack et Liebeskind mentionnent: "Nous sommes dégoûtés du fait que la douleur est moins bien soulagée chez les personnes les plus vulnérables, soit les enfants et les personnes âgées.» Cet éditorial a été écrit il y a 15 ans; depuis ce temps, de nombreux travaux ont été réalisés en ce qui a trait à la douleur chez l'enfant. Cependant, en ce qui concerne les personnes âgées, le soulagement de la douleur demeure une préoccupation de second plan (Gibson, 1998), surtout au regard des personnes âgées qui sont atteintes de déficits cognitifs et qui peuvent difficilement exprimer leur douleur. L'incidence de la douleur chez les personnes âgées atteintes de déficits cognitifs est difficile à établir. Une seule étude, celle de Ferrell, Ferrell et 


\section{au petit matin comme une fresque}

du dessous vient une crevasse

\author{
elle s'écaille \\ elle s'écroule
}

la vie

Rivera (1995) réalisée auprès de 217 bénéficiaires âgés atteints de déficits cognitifs rapporte une incidence de douleur de 62\%. Marzinski (1991) croit que les personnes âgées démentes éprouvent de la douleur, cependant, comme elles ne peuvent la verbaliser, leur douleur est souvent ignorée. À cet égard, l'étude corrélationnelle de Horgas et Tsais (1998), qui regroupait 339 personnes âgées, a démontré que pour des pathologies similaires, les personnes atteintes de déficits cognitifs recevaient significativement moins d'analgésiques, tant en nombre de doses qu'en dosage, que leurs pairs dont les facultés cognitives étaient intactes.

Même si tout porte à croire que les ressources en milieu hospitalier permettent un soulagement optimal de la douleur, ceci n'est pas nécessairement le cas. Au contraire, plusieurs études ont démontré que la douleur est généralement mal contrôlée à l'hôpital (Carr, 1990; Closs, 1994; Melzack et Liebeskind, 1987). Les facteurs faisant obstacles à l'évaluation et au soulagement de la douleur chez les personnes âgées ont été identifiés par plusieurs auteurs. Ils se regroupent en deux catégories, ceux liés à la personne âgée elle-même et ceux relevant du personnel soignant.

Parmi les facteurs liés à la personne âgée elle-même, il y a:

1. Les déficits cognitifs qui rendent difficile l'évaluation de la douleur. La plupart des auteurs (Closs, 1996; Hayes, 1995; Hurley et al., 1992) s'entendent pour affirmer que seule l'observation des comportements des personnes atteintes de déficits cognitifs pourraient fournir aux soignants de l'information sur la présence de douleur.

2. L'attitude des personnes âgées envers la douleur. En effet, celles-ci ont tendance à ne pas signaler leur douleur au personnel soignant par crainte des

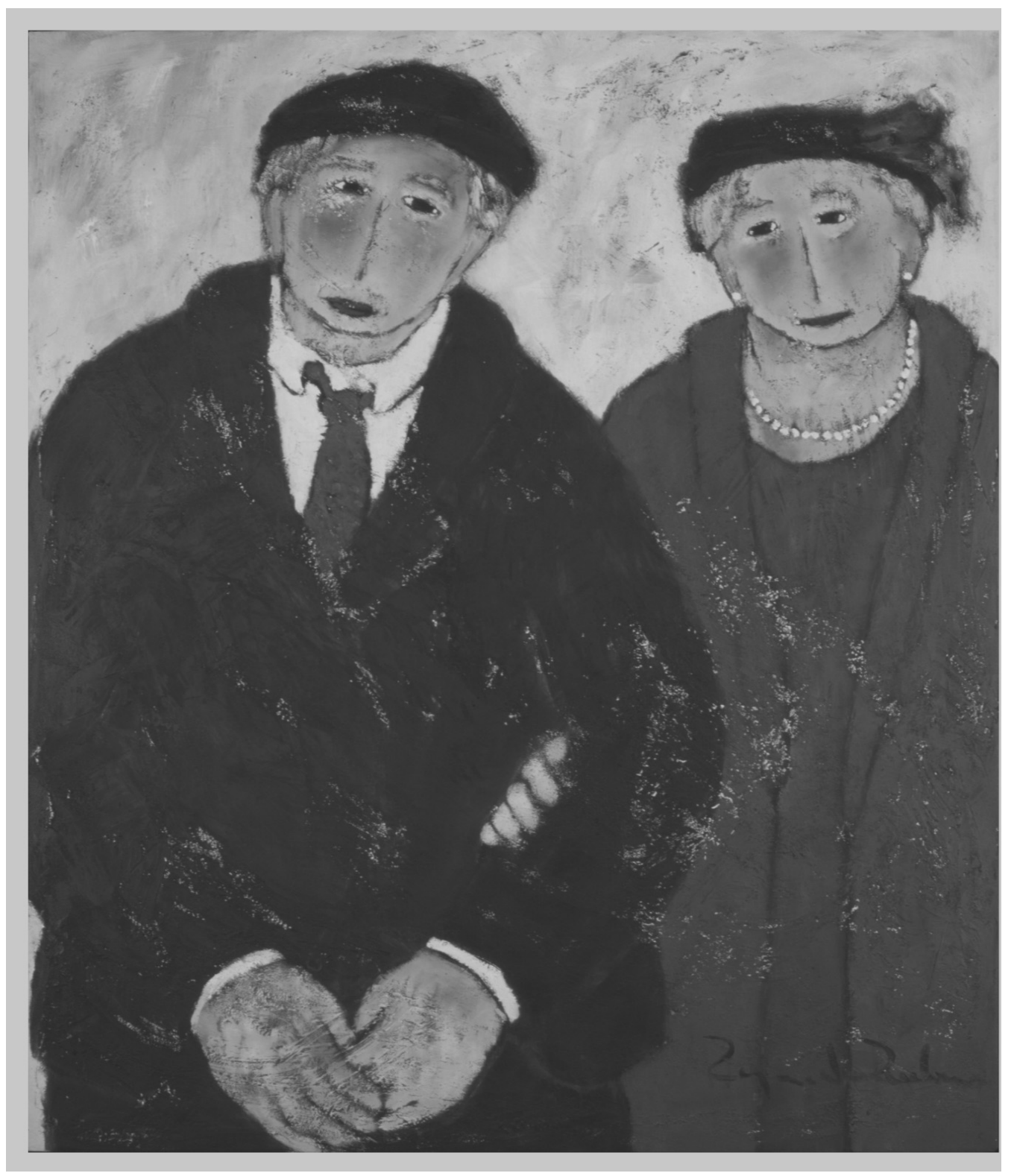

conséquences. Se plaindre de douleur peut impliquer des tests et des procédures diagnostics, des rendez-vous, des hospitalisations, des chirurgies, des changements de médicaments, etc. Certaines personnes âgées conçoivent la douleur comme étant une conséquence normale reliée à l'âge. D'autres, soit à cause de leur culture ou de leurs convictions religieuses, croient qu'il est inacceptable de se plaindre et qu'il vaut mieux apprendre à vivre silencieusement avec la douleur.

3. La polypharmacologie caractéristique des personnes âgées, de même que la coexistence de maladies chroniques et aiguës rendent plus complexe la planification de l'analgésie. De même certains changements physiologiques liés à l'âge peuvent interférer avec l'effet des analgésiques (Closs, 1994, 1996; Gibson, 1998; Gloth, 2000; Herr et Mobily, 1991;
Marzinski, 1991; McCaffery, 1999;

Parke, 1998; Ryan et al., 1994).

Le manque de connaissances ainsi que les mythes et les stéréotypes à l'égard de la douleur des personnes âgées sont les principaux obstacles, chez le personnel soignant, à l'évaluation et au soulagement de la douleur (Bergh et Sjöström, 1999; Gibson, 1998; McCaffery, 1999; Parke, 1998).

Après avoir passé en revue huit manuels traitant des soins infirmiers auprès des personnes âgées, cumulant plus de 5000 pages de texte, Ferrell et Ferrell (1990) n'ont relevé que 18 pages portant sur le soulagement de la douleur. Ceci témoigne de la pauvreté de l'intérêt et du corpus de connaissances à l'égard de la douleur chez la personne âgée.

De plus, dans une étude menée auprès de 208 infirmières, Closs (1996) a démontré que les infirmières des unités de chirurgie possédaient de meilleures connaissances des particularités de la douleur chez la 
personne âgée que les infirmières des unités de gériatrie. Plusieurs infirmières entretenaient de fausses croyances concernant le traitement pharmacologique de la douleur et exagéraient le risque de détresse respiratoire causé par les opioïdes. De plus, un tiers des participantes à l'étude ont mentionné que la douleur et l'inconfort étaient des conséquences normales du vieillissement.

Plusieurs des infirmières participant à cette étude ont mentionné avoir utilisé des méthodes alternatives pour soulager la douleur des personnes âgées et plusieurs se disaient intéressées à perfectionner leurs habiletés dans ce domaine. Closs (1996) n'a pas identifié de disparité dans les connaissances des infirmières selon leur scolarité, leurs années d'expérience et leur quart de travail.

Dans l'étude de Ryan et al. (1994), les connaissances et les attitudes concernant la douleur cancéreuse des infirmières travaillant en oncologie sont comparées à celles des infirmières travaillant en soins de longue durée. Les résultats de cette étude ont démontré que les infirmières en oncologie possédaient une meilleure connaissance du contrôle pharmacologique de la douleur cancéreuse. Cependant, les deux groupes d'infirmières ont obtenu de piètres résultats au questionnaire concernant les opioïdes. Un résultat troublant, car les infirmières travaillant en soins de longue durée croient que les bénéficiaires exagèrent leur douleur. Ces infirmières croient que le quart de leurs bénéficiaires, souffrant de cancer, amplifie l'intensité de leur douleur. Sous l'emprise de ces préjugés, ces infirmières nient un principe fondamental de l'évaluation de la douleur qui est de croire en l'évaluation de la douleur faite par le bénéficiaire qui souffre (McCaffery, 1999).

Bergh et Sjöström (1999) mentionnent que pour améliorer le soulagement de la douleur chez les personnes âgées, les infirmières doivent accroître leurs connaissances et modifier leurs pratiques à l'égard du contrôle de la douleur. De l'avis de ces auteurs, il est très important que les infirmières actualisent leurs connaissances sur la douleur. Leur étude comparative portant sur l'évaluation de la douleur démontre une déficience importante de la formation reçue sur la douleur. Une façon, selon eux, d'augmenter les connaissances et de changer les pratiques cliniques liées au contrôle de la douleur est d'utiliser les nouvelles connaissances issues de la recherche.

L'étude de Ryan et al. (1994) a, pour sa part, identifié chez les infirmières une readiness pour l'apprentissage au regard du soulagement de la douleur. En effet, 75\% des infirmières de cette étude ont ciblé le manque de connaissances comme étant un obstacle au soulagement de la douleur. Ceci démontre que les infirmières évaluent de
UN RÉSULTAT TROUBLANT,

CAR LES INFIRMIÈRES TRAVAILLANT EN SOINS DE LONGUE DURÉE CROIENT QUE LES BÉNÉFICIAIRES EXAGÈRENT LEUR DOULEUR. façon réaliste leur besoin d'améliorer leurs connaissances et leurs habiletés au regard du soulagement de la douleur.

À l'Hôpital Sainte-Anne (HSA) de Sainte-Anne-de-Bellevue au Québec, le soulagement de la douleur des bénéficiaires âgés préoccupe grandement les infirmières. Plus de 130 infirmières (environ 85\%) ont participé récemment à des séances de formation portant sur des outils d'évaluation de la douleur. Lors de ces séances, les infirmières ont exprimé leur besoin d'approfondir leurs connaissances au regard de l'évaluation de la douleur (particulièrement auprès des bénéficiaires atteints de déficits cognitifs), de la pharmacologie et des méthodes alternatives au soulagement de la douleur. Les chercheurs ont également été en mesure de constater, lors de ces séances, que certains préjugés persistaient en lien avec la douleur chez la personne âgée.

$\mathrm{Au}$ Québec, les programmes de formation en sciences infirmières portant sur l'évaluation et le soulagement de la douleur chez la personne âgée sont rarissimes et ceux existants ne sont pas issus d'un processus de recherche. C'est dans cette perspective qu'il nous est apparu utile de mener une étude qualitative de type exploratoire visant à identifier les besoins d'apprentissage, les valeurs et les croyances des infirmières à l'égard de la douleur chez la personne âgée.

Le cadre conceptuel de cette recherche est celui proposé par Loeser et Egan
(1989) modifié par Parke (1998) qui décrit l'expérience de la douleur à travers quatre domaines: nocicepteur ${ }^{1}$, douleur, souffrance et comportements de douleur, et qui intègre l'évaluation de la douleur faite par l'infirmière (figure 1).

Selon ce modèle, l'infirmière entreprend son évaluation à la suite de la manifestation de douleur d'un bénéficiaire. L'évaluation de l'infirmière est teintée de sa formation académique, de ses connaissances théoriques, de ses expériences professionnelles et personnelles, de sa culture, de ses valeurs et ses croyances. Ces variables peuvent influencer positivement ou négativement l'interaction de l'infirmière avec le bénéficiaire souffrant et conséquemment influencer son jugement clinique et son intervention auprès du bénéficiaire. La prémisse de cette recherche repose sur la croyance des chercheurs qu'en agissant sur les connaissances théoriques des infirmières ainsi que leurs valeurs et leurs croyances, le jugement clinique et, subséquemment, les interventions de celles-ci seront modifiés positivement. Ceci devrait produire comme résultat un meilleur soulagement de la douleur du bénéficiaire souffrant.

\section{OBJECTIF DE L'ÉTUDE}

Identifier les besoins d'apprentissage, les valeurs et les croyances d'infirmières à l'égard de la douleur chez la personne âgée afin de développer un programme de formation adapté.

\section{FIGURE 1 - EXPÉRIENCE DE LA DOULEUR (adapté de Parke, 1998, p. 22)}

\begin{tabular}{|c|c|c|}
\hline $\begin{array}{l}\text { Éducation/formation } \\
\text { Connaissances théoriques }\end{array}$ & $\begin{array}{r}\text { INFIRMIÈRE } \\
\downarrow \\
\text { tements de douleur } \\
\text { Souffrance } \\
\text { Douleur } \\
\text { Nocicepteur } \\
\text { ÉRIENCE DE LA I }\end{array}$ & $\begin{array}{c}\text { Expérience personnelle } \\
\text { Expérience professionnelle } \\
\text { Valeurs/croyances/culture }\end{array}$ \\
\hline
\end{tabular}




\section{PHASES DE LA RECHERCHE}

L'étude actuelle représente la première étape d'un projet de recherche de trois phases (tableau 1). Au cours de cette première phase, les chercheurs ont eu recours à des groupes de discussion pour cerner les connaissances et les besoins des infirmières en ce qui a trait à la gestion de la douleur chez les personnes âgées. Ils ont également obtenu des renseignements sur les valeurs et les croyances des infirmières à cet égard. La deuxième phase de l'étude prévoit principalement l'élaboration et la mise en œuvre d'un programme de formation sur la gestion de la douleur chez les personnes âgées, y compris une évaluation des connaissances de base des infirmières et des connaissances acquises grâce aux séances de formation. Au cours de la troisième phase, les chercheurs comptent évaluer l'impact des séances de sensibilisation par un post-test et un examen des notes rédigées par les infirmières sur la gestion de la douleur.

\section{MILIEU DE L'ÉTUDE}

L'étude a été réalisée à l'Hôpital SainteAnne, établissement fédéral offrant des soins et un hébergement à 551 anciens combattants de la Première Guerre mondiale, de la Seconde Guerre mondiale et de la guerre de Corée.

\section{DEVIS DE L'ÉTUDE}

Les chercheurs ont adopté une approche qualitative pour cette étude exploratoire, parce qu'ils voulaient décrire les besoins en apprentissage, les valeurs et les croyances des infirmières dans le but d'obtenir des données factuelles. Les recherches qualitatives sont particulièrement utiles pour l'étude de phénomènes ou d'événements mal connus (Appleton, 1995; Field et Morse, 1985). Elles mettent l'accent sur les expériences personnelles et peuvent donner lieu à des descriptions riches et détaillées de phénomènes encore inexplorés (Appleton, 1995). Cette approche se révèle donc très utile pour les chercheurs.

\section{ÉCHANTILLON}

Les chercheurs ont sélectionné de façon aléatoire un échantillon de 45 infirmières soignantes faisant partie du personnel infirmier de l'hôpital. Huit infirmières gestionnaires ont été invitées à prendre part à l'étude, mais leur groupe a été interrogé séparément et elles n'ont pas participé aux discussions avec les infirmières soignantes. L'échantillon représentait environ 30\% de l'effectif total des infirmières de l'Hôpital Sainte-Anne. Les participantes ont été divisées en deux groupes, le premier composé de celles qui comptaient moins de 10 ans

TABLEAU 1 - PHASES DE LA RECHERCHE

\begin{tabular}{|l|l|l|l|}
\hline & $\begin{array}{l}\text { PHASE 1 } \\
\text { Exploration et } \\
\text { développement }\end{array}$ & $\begin{array}{l}\text { PHASE 2 } \\
\text { Mise en œuvre } \\
\text { d'un programme } \\
\text { de formation }\end{array}$ & $\begin{array}{l}\text { PHASE 3 } \\
\text { Évaluation }\end{array}$ \\
\hline $\begin{array}{l}\text { Type } \\
\text { d'étude }\end{array}$ & $\begin{array}{l}\text { Étude } \\
\text { exploratoire/ } \\
\text { descriptive. }\end{array}$ & $\begin{array}{l}\text { Étude comparative } \\
\text { (avant et après les } \\
\text { séances de } \\
\text { formation). }\end{array}$ & Étude évaluative. \\
\hline Méthodologie & $\begin{array}{l}\text { Groupes de } \\
\text { discussion } \\
\text { (infirmières } \\
\text { soignantes, } \\
\text { infirmières } \\
\text { gestionnaires et } \\
\text { personnes âgées). }\end{array}$ & $\begin{array}{l}\text { Séances de } \\
\text { sensibilisation } \\
\text { (modules de } \\
\text { cyberapprentissage), } \\
\text { prétest (T-1) et } \\
\text { post-test (T-2) sur } \\
\text { les connaissances } \\
\text { concernant } \\
\text { la douleur. }\end{array}$ & $\begin{array}{l}\text { Examen des notes des } \\
\text { infirmières et évaluation des } \\
\text { connaissances (post-test) [T-3]. }\end{array}$ \\
\hline Objectifs & $\begin{array}{l}\text { Évaluation des } \\
\text { besoins, des } \\
\text { valeurs et des } \\
\text { croyances des } \\
\text { infirmières. }\end{array}$ & $\begin{array}{l}\text { Évaluation } \\
\text { et acquisition } \\
\text { des connaissances. }\end{array}$ & $\begin{array}{l}\text { Évaluation de l'impact } \\
\text { cliniques en gestion de la } \\
\text { douleur et sur l'intégration } \\
\text { des connaissances } \\
\text { par les infirmières. }\end{array}$ \\
\hline Stratégie & $\begin{array}{l}\text { Élaboration } \\
\text { d'un programme } \\
\text { de formation. }\end{array}$ & $\begin{array}{l}\text { Suivi des résultats } \\
\text { chez les infirmières. }\end{array}$ & $\begin{array}{l}\text { Suivi des résultats chez les } \\
\text { patients et les infirmières. }\end{array}$ \\
\hline
\end{tabular}

d'expérience, et le second, du personnel comptant plus de 10 ans d'expérience. Pour favoriser la discussion, les chercheurs ont créé des groupes de discussion composés chacun de six infirmières en moyenne.

En tout, 31 infirmières en soins primaires (69\%) et six infirmières gestionnaires (75\%) ont participé aux groupes de discussion. La majorité des infirmières $(\mathrm{N}: 38)$ avaient plus de 10 ans d'expérience en soins infirmiers. Seules six infirmières comptaient moins de 10 ans d'expérience dans le domaine.

\section{QUESTIONNAIRES}

Afin de diriger les entrevues avec les groupes de discussion, les chercheurs ont eu recours à un questionnaire structuré que l'une d'elles avait préparé à la lumière de la documentation consultée et de son expérience professionnelle auprès de la clientèle concernée. Ce questionnaire comportait 10 questions ouvertes sur les trois grandes variables suivantes: les besoins en apprentissage (quatre questions), les valeurs (deux questions) et les croyances (quatre questions). Voici des exemples de questions figurant dans le questionnaire:

- Besoins en apprentissage: Décrivez le rôle des infirmières dans l'évaluation et le contrôle de la douleur.

- Valeurs: Quel est l'élément qui revêt le plus d'importance pour vous lorsque vous dispensez des soins à vos patients âgés?
- Croyances: Que pensez-vous de l'utilisation de narcotiques comme méthode de traitement de la douleur chez les patients âgés?

Les chercheurs ont également mesuré les besoins en apprentissage au moyen du questionnaire sur la gestion de la douleur de McCaffery et Pasero (1999). Ce questionnaire renferme 20 questions dichotomisées (vrai ou faux) qui mesurent les connaissances de base sur la gestion de la douleur. Le score obtenu peut varier de 0 à 20 . Plus le score est élevé, plus les pratiques de gestion de la douleur sont adéquates. La formule de Kuder-Richardson, qui a été appliquée pour mesurer la cohérence interne de l'échelle, a produit un coefficient de 0,62 .

Trente-sept infirmières ont répondu au questionnaire sur la gestion de la douleur. Leur score moyen s'élevait à 9,35 $\pm 1,69$. Les questions liées aux connaissances pharmacologiques ont contribué à faire diminuer le score.

\section{MÉTHODE DE COLLECTE DES DONNÉES (groupes de discussion)}

Les infirmières ont toutes reçu une lettre faisant état des objectifs de l'étude et leur demandant de participer à une discussion en groupe (focus group) à une date et une heure prédéterminées.

Les réunions de groupe étaient animées par les deux chercheurs principaux 
et duraient un maximum de 60 minutes. Les infirmières y ont assisté pendant leurs heures de travail. Les chercheurs se sont servis du questionnaire présenté pour structurer la discussion et pour éliminer tout biais dans les groupes de discussion. Les questions ouvertes encouragent les sujets à parler en détail de leurs propres expériences (Appleton, 1995). Les discussions ont été enregistrées sur cassette, puis transcrites à des fins d'analyse. Avant la fin de chaque séance, l'un des chercheurs demandait aux participantes de remplir le questionnaire de McCaffery sur la gestion de la douleur. Les réponses fournies sur les cassettes et sur les questionnaires sont demeurées anonymes en tout temps.

\section{ANALYSE DES DONNÉES}

Les données obtenues avec le questionnaire sur la gestion de la douleur ont été analysées au moyen du logiciel SAS.

Le contenu des entrevues a été soumis à la méthode d'analyse décrite par Miles et Huberman (1984). Cette méthode prévoit la réduction et la représentation graphique des données.

\section{RÉDUCTION DES DONNÉES}

Le terme réduction des données désigne le processus consistant à sélectionner, à concentrer, à simplifier, à résumer et à transformer des données (Miles et Huberman, 1994), afin de tirer une signification et une synthèse des mots employés par les répondants (Marshall et Rossman, 1989).

Une secrétaire de recherche a écouté les cassettes et en a transcrit le contenu en numérotant chaque ligne. Au début du processus de réduction des données, les chercheurs ont codé chaque ligne ou groupe de lignes qui représentait un thème. Ils ont codé, de façon indépendante, les transcriptions des trois premières interviews avant de comparer leurs résultats. Un accord à $90 \%$ a été obtenu sur la codification des thèmes attribués. Après le codage, les transcriptions de l'ensemble des entrevues ont été analysées par le logiciel d'analyse qualitative N'VIVO. Les lignes ou groupes de lignes liés à chaque thème ont ensuite été réunis. Ce travail d'établissement de catégories s'appelle "analyse du contenu latent» (Field et Morse, 1985). De l'analyse des données sont ressortis huit principaux thèmes. Les voici: besoins en apprentissage, croyances, valeurs, communication avec les familles, organisation du travail, communication avec les patients, communication avec les médecins et relations professionnelles entre les infirmières. Les chercheurs ont décidé de présenter uniquement les données liées aux trois thèmes qui revêtaient le plus d'intérêt pour leur étude, à savoir les besoins en apprentissage, les croyances et les valeurs du personnel infirmier. Enfin, deux infirmières qui avaient participé aux groupes de discussion ont réalisé un examen indépendant des données réduites, et toutes deux ont approuvé les résultats présentés aux tableaux 2 et 3 .

\section{CROYANCES}

Les croyances sont définies comme étant des configurations cognitives que se forment les individus ou que partagent les personnes d'une même culture. Ce sont les croyances qui donnent leur signification aux événements vécus (Lazarus et Folkman, 1984).

Le tableau 2 présente les principales croyances au sujet de la gestion de la douleur qui ont été mentionnées par les infirmières au cours des entrevues.

\section{VALEURS}

Les valeurs concernent ce qui est vrai, beau ou bien, selon un jugement personnel plus ou moins en accord avec celui de la société de l'époque (Le Petit Robert, 1993). Voici les trois principales valeurs qui ont été cernées par les infirmières au cours des entrevues: 1) assurer le confort du patient; 2) respecter les souhaits du patient; 3) promouvoir la qualité de vie.

Le tableau 3 présente les principaux besoins en apprentissage cernés par les infirmières dans leurs discussions.

\section{DISCUSSION}

Les conclusions tirées de cette étude viennent corroborer les résultats de recherches antérieures (Ersek et al., 2000; Herr, 2002), qui révèlent de grandes lacunes sur le plan des connaissances en pharmacologie chez les infirmières. Dans la présente étude, les infirmières ont presque été unanimes à dire qu'elles n'avaient pas assez de connaissances en pharmacologie. Cette affirmation a été corroborée par certaines fausses croyances qu'entretiennent les infirmières au sujet de la gestion de la douleur chez les personnes âgées, notamment: de fausses conceptions au sujet des médicaments PRN (Pro Re Nata - au besoin), la crainte d'administrer la dernière dose et de fausses conceptions au sujet de l'administration de narcotiques aux personnes âgées. Les médicaments PRN représentent un défi particulier pour les infirmières. Il ne semble exister aucun consensus sur la façon de les administrer. Devraient-ils être offerts régulièrement, administrés au besoin ou de façon régulière?

$\mathrm{Au}$ cours des discussions, il est ressorti que le manque de connaissances en pharmacologie était également une source de frustration pour les infirmières dans leurs communications avec les médecins. En effet, de nombreuses participantes ont indiqué ne pas approuver l'administration d'analgésiques prescrits, mais être incapables d'en discuter avec le médecin en raison d'un manque de connaissances. Cette situation explique pourquoi les infirmières estiment devoir bâtir une relation de confiance et adopter de meilleures stratégies de communication avec les médecins. Il a également été mentionné que le fait de participer à des séances de sensibilisation communes sur la

\section{TABLEAU 2 - PRINCIPALES CROYANCES MENTIONNÉES PAR LES INFIRMIÈRES}

\begin{tabular}{|c|c|c|c|c|}
\hline $\begin{array}{l}\text { Croyances } \\
\text { concernant } \\
\text { les médicaments }\end{array}$ & $\begin{array}{l}\text { Croyances } \\
\text { concernant } \\
\text { la gestion } \\
\text { de la douleur }\end{array}$ & $\begin{array}{l}\text { Croyances } \\
\text { des familles } \\
\text { selon les } \\
\text { infirmières }\end{array}$ & $\begin{array}{l}\text { Croyances } \\
\text { des patients } \\
\text { selon les } \\
\text { infirmières }\end{array}$ & $\begin{array}{l}\text { Croyances } \\
\text { des médecins } \\
\text { selon les } \\
\text { infirmières }\end{array}$ \\
\hline $\begin{array}{l}\text { Fausses conceptions } \\
\text { liées à } \\
\text { l'administration } \\
\text { de narcotiques }\end{array}$ & $\begin{array}{l}\text { Absence de } \\
\text { douleur (possible } \\
\text { ou impossible) }\end{array}$ & $\begin{array}{l}\text { Peur de la } \\
\text { morphine }\end{array}$ & $\begin{array}{l}\text { Peur de la } \\
\text { dépendance } \\
\text { aux narcotiques }\end{array}$ & $\begin{array}{l}\text { Peur des } \\
\text { narcotiques }\end{array}$ \\
\hline $\begin{array}{l}\text { Fausses conceptions } \\
\text { liées aux } \\
\text { médicaments PRN }\end{array}$ & $\begin{array}{l}\text { Maintien de la } \\
\text { qualité de vie }\end{array}$ & & $\begin{array}{l}\text { Souffrance tolérée } \\
\text { en raison des } \\
\text { croyances } \\
\text { religieuses }\end{array}$ & $\begin{array}{l}\text { Peur de la } \\
\text { dépression } \\
\text { respiratoire }\end{array}$ \\
\hline $\begin{array}{l}\text { Peur d'administrer } \\
\text { la dernière dose }\end{array}$ & $\begin{array}{l}\text { Respect de ce que } \\
\text { dit le patient au } \\
\text { sujet de sa propre } \\
\text { douleur }\end{array}$ & & $\begin{array}{l}\text { Peur de prendre } \\
\text { davantage de } \\
\text { médicaments }\end{array}$ & \\
\hline $\begin{array}{l}\text { Fausses conceptions } \\
\text { concernant } \\
\text { l'évocation } \\
\text { de la douleur } \\
\text { par les patients }\end{array}$ & & & & \\
\hline
\end{tabular}




\begin{tabular}{|c|c|c|c|c|c|}
\hline $\begin{array}{l}\text { Connaissances en } \\
\text { pharmacologie }\end{array}$ & $\begin{array}{l}\text { Évaluation } \\
\text { de la douleur }\end{array}$ & $\begin{array}{l}\text { Pathophysiologie } \\
\text { de la douleur }\end{array}$ & $\begin{array}{l}\text { Méthodes non } \\
\text { pharmacologiques }\end{array}$ & Communication & $\begin{array}{l}\text { Évaluation de la } \\
\text { douleur chez les } \\
\text { patients souffrant } \\
\text { de déficience } \\
\text { cognitive }\end{array}$ \\
\hline $\begin{array}{l}\text { Connaissance } \\
\text { des analgésiques }\end{array}$ & Échelles à utiliser & $\begin{array}{l}\text { Neurophysiologie } \\
\text { de la douleur }\end{array}$ & $\begin{array}{l}\text { Contribution } \\
\text { des autres } \\
\text { professionnels }\end{array}$ & $\begin{array}{l}\text { Stratégie pour } \\
\text { l'échange } \\
\text { d'information } \\
\text { avec les médecins }\end{array}$ & $\begin{array}{l}\text { Échelles propres } \\
\text { à cette clientèle }\end{array}$ \\
\hline $\begin{array}{l}\text { Connaissance } \\
\text { des médicaments } \\
\text { PRN }\end{array}$ & $\begin{array}{l}\text { Signes et symptômes } \\
\text { de la douleur }\end{array}$ & Types de douleur & $\begin{array}{l}\text { Soins } \\
\text { aux personnes } \\
\text { en fin de vie }\end{array}$ & $\begin{array}{l}\text { Stratégies pour } \\
\text { promouvoir l'échange } \\
\text { de connaissances } \\
\text { entre membres } \\
\text { de l'équipe }\end{array}$ & $\begin{array}{l}\text { Connaissance } \\
\text { du contexte } \\
\text { des soins }\end{array}$ \\
\hline \multirow[t]{2}{*}{$\begin{array}{l}\text { Connaissance } \\
\text { des médicaments } \\
\text { souvent prescrits } \\
\text { aux personnes âgées }\end{array}$} & Types de douleur & $\begin{array}{l}\text { Impact de la } \\
\text { comorbidité } \\
\text { sur la douleur }\end{array}$ & $\begin{array}{l}\text { Thérapies issues } \\
\text { de la médecine } \\
\text { douce }\end{array}$ & $\begin{array}{l}\text { Stratégies pour mieux } \\
\text { communiquer avec } \\
\text { les médecins et établir } \\
\text { une relation } \\
\text { de confiance }\end{array}$ & \\
\hline & & & & $\begin{array}{l}\text { Participation à la } \\
\text { formation sur la } \\
\text { douleur donnée } \\
\text { aux médecins }\end{array}$ & \\
\hline
\end{tabular}

douleur avec les médecins et les membres de l'équipe multidisciplinaire contribuerait à une plus grande cohésion dans la gestion de la douleur des patients. Parmi les autres besoins en apprentissage cernés, il convient de mentionner la connaissance des différents types de douleur et l'évaluation de la douleur, surtout chez les patients souffrant de déficience cognitive. Cette situation demeure d'ailleurs un défi clinique, comme le mentionne souvent la documentation sur le sujet.

Dans l'ensemble, les soins que dispensent les infirmières aux patients âgés sont fondés sur un ensemble de valeurs solidement ancrées. Cependant, ces valeurs semblent entrer en contradiction avec certaines de leurs croyances (fausses conceptions). En effet, plusieurs participantes ont dit ne pas croire à la douleur de certains patients, surtout ceux qui ont des troubles psychiatriques, et bon nombre d'entre elles ont qualifié ces patients de manipulateurs à la recherche d'attention.

Les chercheurs ont été impressionnés par le fait que, lorsqu'elles ont été interrogées au sujet de leurs valeurs, les infirmières ne s'en sont pas tenues à l'aspect physique des soins. Elles ont plutôt fait référence à des concepts beaucoup plus larges et multifactoriels, entre autres: assurer le confort des patients, respecter leurs souhaits et promouvoir la qualité de vie.

En ce qui a trait aux croyances des infirmières, la crainte d'administrer la dernière dose est encore très répandue, malgré les nombreux efforts déployés pour les sensibi- liser et pour éliminer cette croyance erronée. C'est là un autre exemple qui prouve que les connaissances purement théoriques ne sont pas suffisantes pour modifier des pratiques cliniques. Il faut travailler à ébranler les croyances qui sont souvent des obstacles majeurs à une gestion adéquate de la douleur des personnes âgées.

Par ailleurs, les infirmières ont des opinions assez partagées sur la possibilité de soulager complètement la douleur. Certaines déclarent qu'il est possible d'y arriver, tandis que d'autres prétendent qu'il s'agit d'un objectif irréaliste. D'autres encore affirment qu'il importe davantage de faire en sorte que le patient demeure alerte et fonctionnel que de le soulager complètement de sa douleur.

La principale croyance des familles est une fausse conception liée à la crainte de la morphine. Selon les infirmières, les familles associent souvent la morphine à un décès imminent et refusent parfois que ce médicament soit utilisé, de peur qu'il ne précipite la mort de leur proche.

Quant aux patients, il semble que leur principale préoccupation soit, de l'avis des infirmières, la peur de développer une dépendance à l'égard des médicaments administrés. Bon nombre d'entre eux refusent en effet de prendre quelque médicament que ce soit, de crainte d'en devenir dépendants. Toujours selon les infirmières ayant participé à l'étude, certains patients hésitent à se plaindre de douleurs parce qu'ils croient que la souffrance les aidera à expier leurs péchés et leur assurera une place au ciel. Ces propos sont corroborés par la documentation consultée.

Compte tenu des craintes et des fausses conceptions des infirmières concernant le recours à des narcotiques, il n'est pas étonnant que les familles et les patients qui dépendent des professionnels pour l'obtention d'information et de soins nourrissent eux aussi des craintes et des fausses conceptions au sujet des narcotiques, en particulier la morphine. On peut penser que l'information fournie aux patients et aux familles sera sans doute conditionnée par les fausses conceptions et les craintes des professionnels qui participent à la prestation des soins.

Cette étude a fait ressortir la nécessité impérieuse de donner aux infirmières une formation en pharmacologie dans le domaine du contrôle de la douleur. Elle a également mis en évidence la nécessité de donner aux patients et aux familles suffisamment d'information sur l'administration de médicaments, en particulier sur l'usage thérapeutique des narcotiques comme la morphine. Cependant, la formation ne doit pas se limiter au contenu purement théorique. Afin de susciter un changement dans les pratiques cliniques, il faut travailler sur les croyances et les préjugés du personnel infirmier relativement à la gestion de la douleur des personnes âgées. Ce sont souvent des obstacles importants à une gestion adéquate de la douleur. 


\section{Bibliographie}

APPLETON, J.V. (1995). "Analysing qualitative interview data: addressing issues of validity and reliability », Journal of Advanced Nursing, 22, p. 993-997.

BERGH, I. et B. SJÖSTRÖM (1999). «A comparative study of nurses' and elderly patients' ratings of pain and pain tolerance », Journal of Gerontological Nursing, 25(5), p. 30-36.

BOWLING, A. et P.D. BROWNE (1991). "Social networks, health and emotional well-being among the oldest old in London », Journal of Gerontology, 46(1), p. S20-S32.

CARR, E.C.J. (1990). «Post-operative pain: patients' expectations and experiences", Journal of Advanced Nursing, 15, p. 89-100.

CLOSS, J.S. (1994). "Pain in elderly patients: a neglected phenomenon?», Journal of Advanced Nursing, 19, p. 1072-1081.

CLOSS, J.S. (1996). "Pain and elderly patients: a survey of nurses' knowledge and experiences », Journal of Advanced Nursing, 23, p. 237-242.

EPPS, C.D. (2001). "Recognizing pain in the institutionalized elder with dementia ", Geriatric Nursing, 22(2), p. 71-79.

ERSEK, M., B.M. KRAYBILL et J. HANSBERRY (2000). "Assessing the Educational Needs and Concerns of Nursing Home Staff Regarding End-of-Life Care», Journal of Gerontological Nursing, 26(10), p. 16-26.

FERRELL, B.R. et B.A. FERRELL (1990). «Easing the pain», Geriatric Nursing, 11, p. $175-178$.

FERRELL, B.A., B.R. FERRELL et D. OSTERWEIL (1990). "Pain in the nursing home», Journal of the American Geriatric Society, 38, p. 409-414.

FERRELL, B.A. (1991). «Pain management in elderly people», Journal of the American Geriatric Society, 39, p. 64-73.

FERRELL, B.A., B.R. FERRELL et L. RIVERA (1995). "Pain in cognitively impaired nursing home patients», Journal of Pain and Symptom Management, 10, p. 591-598.

FIELD A.P. et J.M. MORSE (1985), Nursing research: The application of qualitative approaches, Londres, Croom Helm.

FOX, P.L., P. RAINA et A.R. JADAD (1999). «Prevalence and treatment of pain in older adults in nursing homes and other longterm care institutions: a systematic review», Canadian Medical Association Journal, 160(3), p. 329-333.

GIBSON, M.C. (1998). "Improving pain control for the elderly patient with dementia ", American Journal of Alzheimer's Disease, janvier-février, p. 10-14.

GLOTH, M.F. (2000). «Factors that limit pain relief and increase complications », Geriatrics, 55(10), p. 46-52.

HAYES, R. (1995). "Pain assessment in the elderly», British Journal of Nursing, 4, p. $1199-1204$.
HERR, K. (2002). «Chronic pain challenges and assessment strategies », Journal of Gerontological Nursing, 28(1), p. 20-27.

HERR, K.A. et P.R. MOBILY (1991). «Complexities of pain assessment in the elderly: clinical considerations», Journal of Gerontological Nursing, 17(4), p. 12-19.

HORGAS, A.L. et P. TSAIS (1998). "Analgesic drug prescription and use in cognitively impaired nursing home residents ", Nursing Research, 47, p. 235-242.

HURLEY, A.C., B.J. VOLICER, P.A. HANRAHAN, S. HOUDE et L. VOLICER (1992). Assessment of discomfort in advanced Alzheimer patient. Research in Nursing and Health, 15, p. 369-377.

LINDLEY, C.M. et J.A. DALTON (1990).

«Narcotics analgesics», Cancer Nursing, 13, p. 28-38.

LOESER, J.D. et K.J. EGAN (1989). Managing the chronic pain patient: Theory and practice at the University of Washington multidisciplinary pain centre, New York, Raven Press.

MARSHALL, C. et G.B. ROSSMAN (1989). Designing qualitative research, Londres, Sage Publications.

MARZINSKI, L.R. (1991). "The tragedy of dementia: clinically assessing pain in the confused, nonverbal elderly", Journal of Gerontological Nursing, 17(6), p. 25-28.

McCAFFERY, M. (1989). Nursing management of the patient with pain, Philadelphie, Lippincott.

MELZACK, R. et J.C. LIEBESKIND (1987). "The international pain foundation: meeting a need for education in pain management», Pain, 30, p.1-2.

MILES, M.B. et A. HUBERMAN (1984). Qualitative data analysis. A source book of new methods, Londres, Sage Publications.

MILES, M.B. et A. HUBERMAN (1994). An expanded source book. Qualitative data analysis, $2^{\mathrm{e}}$ édition, Thousand Oaks (Californie), Sage Publications.

PARKE, B. (1998). «Gerontological nurses' ways of knowing », Journal of Gerontological Nursing, 24(6), p. 21-28.

RYAN, P., R. VORTHERMS et S. WARD (1994). «Cancer pain: knowledge, attitudes and pharmacologic management », Journal of Gerontological Nursing, janvier, p. 7-15.

UNIVERSITÉ D'OTTAWA et UNIVERSITÉ DE TORONTO (2000). A guide to end-of-life care for seniors, Santé Canada.

YATES, P., A. DEWAR et B. FENTIMAN (1995). "Pain: the views of elderly people living in long-term residential care settings », Journal of Advanced Nursing, 21, p. 667-674.

\section{Note}

1. Nocicepteur: récepteur sensitif captant les messages douloureux. 